\title{
Prevalence De L'anemie Et Des Anticorps Specifiques Anti-P. Falciparum Chez Les PVVIH Et Co-Infectees Par Le Plasmodium Falciparum Au Congo-Brazzaville
}

\section{S. Judith Gbenoudon Satoguina, (PhD)}

Laboratoire d'Immunologie, des Maladies infectieuses et allergiques, ISBA et FAST, Université d’Abomey-Calavi, 04 BP 1221 Cotonou, Benin.

\section{Ruphin Bolanga, (MSc)}

Laboratoire d'Immunologie, des Maladies infectieuses et allergiques, ISBA et FAST, Université d’Abomey-Calavi, 04 BP 1221 Cotonou, Benin.

Laboratoire National de Santé Publique, Université Marien Ngouabi, Congo

Laboratoire de Microbiologie appliquée, Université de Lomé, Togo

Etienne Mokondjimobe, (MD)

Laboratoire National de Santé Publique, Université Marien Ngouabi, Congo

Comlan De Souza, (PhD, Prof.)

Laboratoire National de Santé Publique, Université Marien Ngouabi, Congo

doi: 10.19044/esj.2016.v12n33p124 URL:http://dx.doi.org/10.19044/esj.2016.v12n33p124

\begin{abstract}
HIV and P. falciparum lead to diseases with overlapping endemic areas. Both diseases are leading cause of nearly 2 millions of deaths each year, which occur mostly in developing countries. People living with HIV are more susceptible to disease progressions. It is therefore important to further understand the biology of HIV and P. falciparum coinfection. The present study sets to characterize the coinfection with regard to malaria specific antibodies and to anemia. We found that antibody and hemoglobin levels as well as CD4 counts differ significantly according to parasite load and to treatment with anti retroviral. People undergoing treatment with antiretroviral, produced specific antimalarial IgG compared to their treatment naïve counterparts.
\end{abstract}

Keywords: Malaria, HIV, Coinfection, Antibody, Anemia

\section{Résumé}

Le VIH et P. falciparum causent tous deux, des maladies qui sont mortelles avec les endémicités qui chevauchent géographiquement. Le paludisme et le VIH causent ensemble près de 2 millions de décès chaque 
année, en majorité dans les pays en voie de développement. Les personnes vivant avec le VIH (PVVIH) présentent une plus grande susceptibilité au paludisme qui augmente dans certains cas le taux de mortalité liée au VIH. Cette étude a eu pour objectifs de caractériser la coinfection VIH et paludisme eu égard á l’anémie, complication commune aux deux maladies, et aux anticorps antipaludiques qui pourraient protéger d'une aggravation liée á la coinfection en neutralisant les parasites. Cette étude a été réalisée au Congo Brazzaville sur des PVVIH sous un traitement antirétroviral et sur des PVVIH non encore sous traitement. Nous avons trouvé que Les taux d'anticorps antipaludiques, de LTCD4+ et d'hémoglobine étaient significativement différents selon que les patients étaient sous ARV ou avaient une goutte épaisse positive. Nous avons conclu, que ces anticorps spécifiques constituent une caractéristique additionnelle des PVVIH qui sont sous un traitement antirétroviral.

Mots clés: Malaria, VIH, Coinfection, Anticorps, Anémie.

\section{Introduction}

Le paludisme, même en déclin, reste à nos jours une maladie lourde de conséquence; les estimations font état de 214 millions d’épisodes palustres en 2015, dont la majorité dans la région Afrique de l’OMS (WHO, 2015). Le nombre de décès dus au paludisme est estimé à 438000 pour l’année 2015 (OMS. 2015 ; Volberding, 2008).

L’infection par le VIH constitue un véritable désastre humain, social et économique en particulier dans les pays les plus pauvres qui sont les plus touchés. C'est en Afrique subsaharienne que l'épidémie est la plus étendue avec plus de 68\% des cas de la planète (Martin-Blondel et al. 2010).

Le paludisme et le VIH causent ensemble près de 2 millions de décès chaque année, en majorité dans les pays en voie de développement, dont 1.2 million pour le compte du VIH seul (ONUSIDA 2015, World Malaria report 2014). Les PVVIH exhibent une plus grande susceptibilité au paludisme qui augmente dans certains cas le taux de mortalité liée au VIH (Renia et al, 2006, Skinner-Adams et al, 2008 ; Alemu et al, 2013). En effet, en prenant des cas d'infections dans une zone hautement endémique au Kenya, il a été établi, que l'infection au VIH et la malaria accroissent le taux de transmission du VIH, par suite d'une élévation de 2 log de la virémie. De même la susceptibilité au paludisme clinique liée á la coinfection serait augmentée (Laith et al, 2006). L’anémie, la diminution du nombre de globule rouge dans le sang et de leur teneur en hémoglobine représente l'une des complications importantes rencontrées lors de l'infection par le VIH (Omoregie et al. 2009 ; Van Geertruyden et al. 2007), mais aussi par P. falciparum. De nombreuses études rapportent une prévalence d'anémie de 
68\% à 95\% chez les patients atteints de SIDA (Oumar et al. 2009 ; D. A. Diallo et al. 2003 ; Laith J. Abu-Raddad et al. 2006). Cette prévalence d’anémie a plusieurs étiologies, parmi lesquelles, le paludisme. En effet l'anémie représente l'une des complications majeures rencontrées lors du paludisme (Sanyalu et al, 2013).

En République du Congo, pour les personnes infectées par le VIH/SIDA, on a une prévalence nationale de 3,2\% (Rapport SEP/CNLS Congo. 2014). Plusieurs études ont été faites en République du Congo sur l'épidémiologie et le phénotypage du VIH (M'PELE et al, Candotti et al. 1999, Bikandou et al. 2000, Niama et al. 2006).

La présente étude a pour but d'évaluer la réponse en anticorps antipaludiques des personnes vivant avec le VIH (PVVIH) sous antirétroviral (ARV) comparées á leurs paires ARV naïves et d’étudier les paramètres biologiques affectés par la coïnfection $\mathrm{Pf} / \mathrm{VIH}$, tels que la charge virale, la parasitémie et le taux d'hémoglobine. Nos résultats indiquent que les anticorps spécifiques contre le Plasmodium falciparum (Pf) sont plus élevés chez les PVVIH sous ARV qui font d'ailleurs moins d'anémie que leurs paires, les PVVIH naïves.

I.

\section{Matériel et Méthodes}

\section{Cadre et type d'étude}

Il s'agit d'une étude prospective-transversale. La collecte d'échantillon s'est faite au Laboratoire National de Santé Publique (LNSP) en R. du Congo de février á aout 2012. Les personnes de sérologie à VIH connue dites naïves et celles sous ARV sont prélevées dans les conditions appropriées au LNSP á Brazzaville au Congo. Les paramètres courants, tels que la goutte épaisse, le frottis, le taux d’hémoglobine, la numération de la formule sanguine, la numération des lymphocytes TCD4+ ont été ensuite mesurés sur place au LNSP en utilisant les automates agréés á cet effet. Le dosage des anticorps IgG totaux contre les antigènes MSP-1 19 de $P$. falciparum a été fait au Laboratoire des maladies infectieuses et allergiques, à l'Institut des Sciences Biomédicales Appliquées (ISBA) en R. du Bénin suivant la procédure décrite ailleurs par Satoguina et al., 2009.

\section{Population d'étude}

Elle était constituée d'un total de 1171 personnes vivant avec le VIH (PVVIH) venant au Laboratoire LNSP dans le cadre du bilan biologique d'Inclusion/Suivi au traitement antirétroviral. Le suivi des patients par les antirétroviraux se faisait selon l'algorithme en vigueur au R. du Congo. Au Congo la combinaison préférentielle en première ligne est basée sur l'association de deux (2) inhibiteurs nucléosidiques de la transcriptase 
inverse (2INTI) et de un (1) inhibiteur non nucléosidique de la transcriptase inverse. Ont été exclues de l'étude toutes les personnes de sérologie VIH connue non inscrites au ARV et les patients ayant reçu une transfusion sanguine dans les trois derniers mois. Pour la présente étude, 746 PVVIH dont tous les paramètres sont au complet ont été retenues, 277 étaient sous ARV et 469 étaient encore naïves au traitement ARV.

\section{Analyses biologiques}

Une goutte épaisse et un frottis étaient faits, selon la méthode décrite précédemment (SATOGUINA et al, 2009). Pour la parasitémie (parasites/ $\square 1$ ), 8000 leucocytes/ $\square 1$ sont considérés. Le sang est prélevé (5 $\mathrm{ml}$ ) dans un tube à EDTA et le comptage des cellules sanguines s'était effectué avec un hémocytomètre (Mindray BC2800, Indonésie). L'anémie a été définie à un taux d'hémoglobine $\leq 11 \mathrm{~g} / \mathrm{dl}$ chez la femme et $\leq 12 \mathrm{~g} / \mathrm{dl}$ chez l'homme (WHO, 2015). La numération des lymphocytes TCD4+ a été faite par tri cellulaire révélé par immunofluorescence (FACS). L'automate utilisé était de type BD FACSCOUNT. Les anticorps antiplasmodiaux ont été dosés par la méthode ELISA indirect comme décrit précédemment (SATOGUINA et al, 2009). Brièvement, les IgG totaux ont été dosés contre la protéine antigénique de surface de mérozoites de $P$. falciparum, le MSP $1_{19}$. Les plasmas utilisés étaient dilués au 1/1000. La révélation est faite avec l'anticorps secondaire conjugué á la peroxydase puis le substrat OPD (l'orthophénylènediamine).

\section{Analyses données}

Les logiciels STATA ${ }^{\circledR}$ version 10 et Graph Pad Prisme version 6 ont été utilisés pour les figures et les tests de comparaison. La valeur de $p<0.05$ est considéré comme significative.

\section{Condition éthique}

Le consentement de chaque patient a été obtenu après une explication détaillée sur la politique nationale de gratuité du suivi biologique des PVVIH, l'importance de ces examens dans la prise des antirétroviraux et l'exploitation de ces résultats à des fins de bon suivi thérapeutique épidémiologique, et de recherche.

\section{Résultats}

\section{La population d'étude fait montre d'une forte prévalence paludique}

Les PVVIH de la présente étude, au nombre de 746 dont 277 PVVIH naïves et 469 PVVIH sous ARV ont été analysées. L’âge moyen de la population d'étude était de $38.68+/-11.16$ ans. La valeur moyenne de la parasitémie était de $201.88+/-747.10$ parasites/ $\mu$ l. Le sexe féminin 
représentait 73,06\% de la population soit 545 PVVIH. La prévalence du paludisme chez les PVVIH dans notre étude était de 22,65\%. La figure 1 indique que les taux de parasitémie sont équivalents pour les deux groupes de patients $(p>0.05)$.

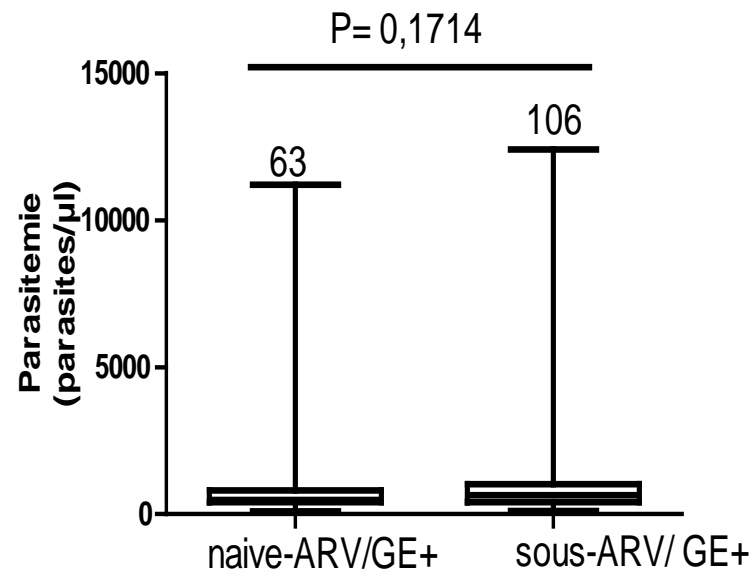

Figure 1: Comparaison de la parasitémie entre les PVVIH naïves et sous ARV.

La parasitémie des PVVIH naïves et sous ARV lu par microscopie n'a pas présenté une différence significative par le test de comparaison de Mann Whitney, $p<0,1714$.

\section{Les PVVIH sont vulnérables á l'anémie qui est corrigée avec l'ARV}

La prévalence de l'anémie chez les PVVIH était de 43,59\% dans l'ensemble soit $61,59 \%$ chez les PVVIH naïves et $43,08 \%$ chez les PVVIH sous ARV. La comparaison des taux d'hémoglobine et des taux de lymphocytes TCD4+ entre les PVVIH naïves et sous ARV avait présenté des différences significatives (Figure 2 et 3 ). Ces résultats confirment que le traitement par les antirétroviraux augmente les taux des LTCD4 et l’hémoglobine. La parasitémie elle même ne présente de corrélation avec aucun autre paramètre biologique; cela pourrait être expliqué par la faible parasitémie au sein de la population d'étude et l'étiologie multiple de certains paramètres comme le taux d’hémoglobine. 


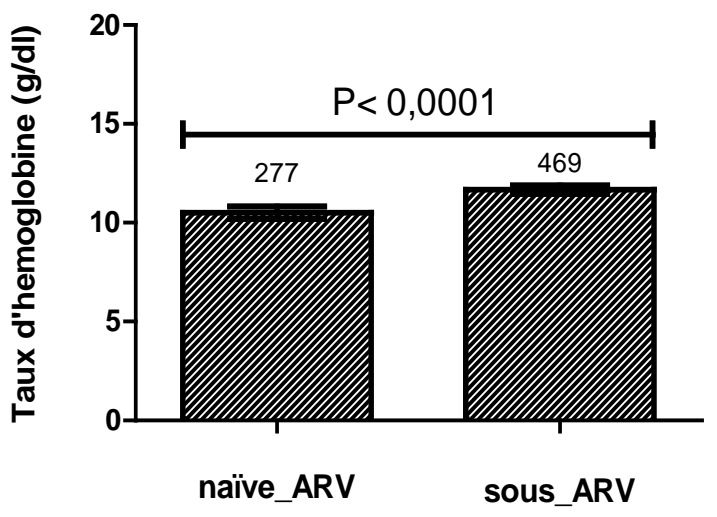

Figure 2: Comparaison des taux d'hémoglobine entre les PVVIH naïves et sous ARV.

Le taux d’hémoglobine des PVVIH naïves et sous ARV présente une différence significative par le test de comparaison de Mann Whitney, $p<0,0001$.

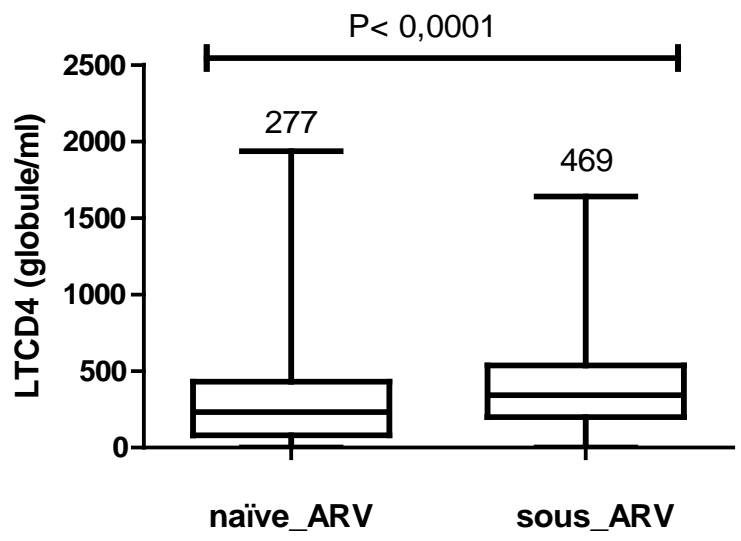

Figure 3: Comparaison des taux de LTCD4 entre les PVVIH naïves et sous ARV. Le taux de LTCD4 des PVVIH naïve et sous ARV présente une différence significative par le test de comparaison de Mann Whitney, $p<0,0001$.

\section{Une réponse anticorps spécifique élevée des PVVIH sous ARV}

Etant en milieu endémique, il était important de savoir si l'ARV augmentait la réponse immunitaire spécifique au paludisme. Nous avons alors dosé les anticorps spécifiques á l'antigène MSP- $1_{19}$ et nous avons vu que les PVVIH sous ARV présentaient des taux plus élevés (Figure 4, $p<0.05$ ) pour ce qui concerne les anticorps IgG totaux spécifiques au paludisme.

Ces résultats montrent que la réponse anti paludique évolue différemment selon que les PVVIH étaient naïves ou sous ARV. 

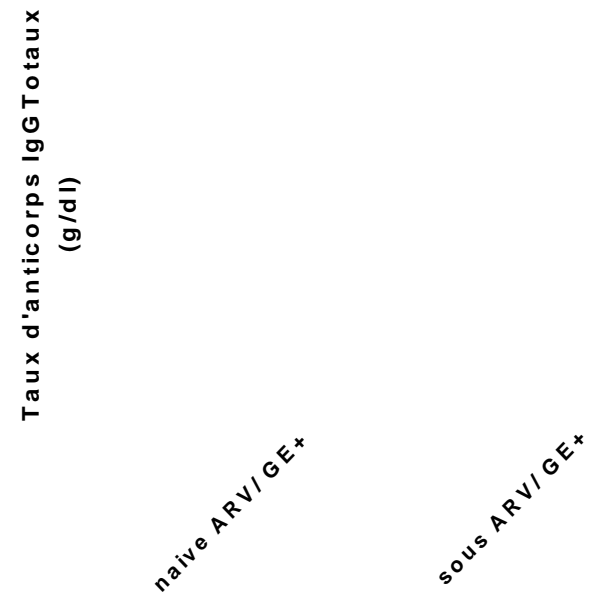

Figure 4: Comparaison des taux de IgG totaux entre les PVVIH naïves et sous ARV de GE +.

Le taux des IgG totaux entre les PVVIH naïves et sous ARV présente une différence significative par le test de comparaison (Student $t$ test), $p=0,048$. Cependant, conformément aux taux de parasitémie, certains PVVIH sous ARV présentaient des anticorps très bas.

Alors que le taux d'hémoglobine ne corrèle significativement ni avec la parasitémie ( $\mathrm{p}=0.46)$, ni avec les anticorps $(\mathrm{p}=0.06)$, comme on pourrait espérer chez les personnes immunocompétentes, ce taux corrèle plutôt bien avec celui des LT CD4 $\left(r^{2}=0.31, p<0.0001\right)$.

\section{Discussions}

Cette étude a eu pour objectifs de caractériser la coinfection VIH et paludisme eu égard á l'anémie, complication commune aux deux maladies, et aux anticorps antipaludiques. C'est une étude qui est d'autant plus importante que les deux maladies sont mortelles et chevauchent géographiquement. La parasitémie 22,65\%, deux fois plus élevée que l'incidence moyenne mondiale (WHO. 2011), indique qu'il s'agit d'un groupe susceptible au paludisme. Cette différence peut s'expliquer par l'effet du VIH sur le paludisme. En effet, le VIH cause l'immunodépression, favorisant l'augmentation de la parasitémie, réciproquement le Plasmodium active les lymphocytes ce qui accroit la multiplication virale (Van Geertruyden et al, 2007).

La répartition par sexe chez les PVVIH se caractérise ici et conformément aux données existantes (ONUSIDA 2012, Omoregie et al, 2009) par une prédominance du sexe féminin sur le sexe masculin, 73,06\% de sexe féminin contre $26.94 \%$ de sexe masculin. La prédominance dans la population d'étude du nombre de PVVIH sous ARV est de 62.87\% PVVIH 
contre 37.13\% PVVIH naïves. Cette prédominance peut s’expliquer par le site de l’étude qui est un centre de suivi biologique des PVVIH.

Les PVVIH sous ARV ont présenté des taux de LTCD4+ moins altérés que les taux des LTCD4+ des PVVIH naïves d'ARV (Figure 3). Cette différence s'explique par l'action des ARV qui ont un mécanisme d'action de protection des LTCD4+ par blocage de l'activité, voire de la réplication virale (Dalakas, 2001).

La prévalence de l'anémie chez les PVVIH dans notre étude est de 43,59\%, soit 313 sur 718 PVVIH. Cette prévalence quoique élevée est inférieure à celle trouvée par Omoregie et al, 2007, soit 60,61\% d'une population mère de 457. Cette différence peut s'expliquer par la définition de l'anémie à un taux d'hémoglobine $\leq 12 \mathrm{~g} / \mathrm{dl}$ chez la femme et $\leq 13 \mathrm{~g} / \mathrm{dl}$ chez l'homme dans leur étude. La prévalence de l'anémie est de 61,59\% chez les PVVIH naïves et de $43,08 \%$ chez les PVVIH sous ARV dans notre étude ; ce résultat corrobore les résultats trouvés dans la Figure 2 qui indique une différence significative entre le taux d'hémoglobine des PVVIH naïves et sous ARV. Il a été très tôt établi que l'anémie est un marqueur indépendant du pronostic de la maladie chez PVVIH (Mocroft, 1989), ainsi donc les ARV, arrêtant la progression de la maladie améliorent l'anémie.

L'absence de différence dans la parasitémie des PVVIH sous ARV naïve (Figure 1) peut s'expliquer par la faible densité parasitaire. En revanche le taux d'anticorps IgG totaux a présenté une différence significative entre PVVIH de goutte épaisse positive naïves et sous ARV (Figure 4). Nous avons aussi une corrélation positive entre le taux d'IgG totaux et le taux de LTCD4+. Le paludisme pourrait bien altérer plus l'état de santé des PVVIH naïves en entrainant ainsi l'anémie et en aggravant l'infection par le VIH.

La faible corrélation entre les taux d'anticorps spécifiques IgG antipaludiques et d'hémoglobine n’exclut pas un rôle de ces anticorps á l'amélioration du taux d'hémoglobine, mais indique l'origine multiple de l'étiologie de l’anémie chez les PVVIH.

\section{Conclusion}

Les taux d'anticorps antipaludiques, de LTCD4+ et d'hémoglobine sont significativement différents selon que les patients étaient sous ARV ou avaient une goutte épaisse positive.

L'anémie était plus fréquente chez les PVVIH naives que chez celles sous ARV, qui d'ailleurs présentent un taux de prévalence d'anticorps IgG antipaludiques plus élevé 


\section{Abréviations}

ARV, Antirétroviraux, PVVIH, Personne vivant avec le VIH, LTCD4, Lymphocytes T CD4.

\section{Remerciements}

Nous remercions le Directeur de LNSP, Mr PARRA Henri Joseph et le Professeur Lamine BABA-MOUSSA pour leur implication personnelle dans cette étude, les participantes et participants de même que le personnel de LNSP pour leur dévouement et la qualité des analyses faites.

\section{References:}

1. Abu-Raddad L. J., Patnaik P., Kublin J.G. (2006). Dual Infection with HIV and Malaria Fuels the Spread of Both Diseases in SubSaharan Africa. Science ; 314, 1603.

2. Akinbo F. O., Omoregie R. (2012). Plasmodium falciparum Infection in HIV-Infected Patients on Highly Active Antiretroviral Therapy (HAART) In Benin City, Nigeria. Journal of Research in Health Sciences; 12, 1.

3. Alemu A., Shiferaw Y., Addis Z., Mathewos B., Birhan W. (2013). Effect of malaria on HIV/AIDS transmission and progression. Parasites \& Vectors; 6:18.

4. Bikandou B., Takehisa J., Mboudjeka I., Ido E., Kuwata T., Miyazaki Y., Moriyama H., Harada Y., Taniguchi Y., Ichimura H., Ikeda M., Ndolo P. J., Nzoukoudi M. Y., M’Vouenze R., M’Pandi M., Parra H. J., M'Pele P., Hayami M. (2000). Genetic subtypes of HIV type 1 in Republic of Congo. AIDS Res. Hum. Retroviruses 16, 613-619.

5. Candotti, D., Tareau, C., Barin, F., Joberty C., Rosenheim M., M’Pele P., Huraux J. M., Agut H. (1999). Genetic subtyping and V3 serotyping of HIV type 1 isolates in Congo. AIDS Res. Hum. Retroviruses 15,309-314.

6. Cohen C., Karstaedt A., Frea00n J. (2005). Increased Prevalence of Severe Malaria in HIV-Infected Adults in South Africa. Clinical Infectious Diseases; 41, 1631-7.

7. Dalakas M.C. (2001). Peripheral neuropathy and antiretroviral drugs. Journal of the Peripheral Nervous System. 6:1; $14-20$.

8. Diallo D. A., Baby M., Dembélé M., Kéita A. (2003). Fréquence, facteurs de risque et valeur pronostique de l'anémie associée au VIH/sida chez l’adulte au Mali. Bull. Soc. Patho. Exot ; 96, 2, 123127.

9. Feiko O. T., Whitworth J. A. G. Malaria and HIV Infection. Prevention and management in resource-poor setting; 5, 66,747-753. 
10. Graeme M. (2002). Anaemia in persons with HIV infection: prognostic marker and contributor to morbidity. AIDS; 4: 13-20

11. GrederBelan A., Chaplain. (2008). Suivi biologique de l’infection à VIH chez l’adulte. Revimmuno-analyse et biologie spécialisée ; 23,96-97

12. Hoffman F. I., Jere C. S., Taylor T. E., Munthaliet P. (1999). The effect of Plasmodium falciparum malaria on HIV-1 RNA blood plasma concentration. AIDS; 13, 4:487-494.

13. Mocroft A., Kirk O., Barton, S., et al., (1999). Anaemia is an independent predictive marker for clinical prognosis in HIV-infected patients from across Europe. AIDS ;13 : 8, 943-950

14. Martin-Blondel G., Soumah M., Camara B., Chabrol A. (2010). Impact du paludisme sur l'infection par le VIH. Médecine et maladies infectieuses ; 40,256-267.

15. Mboudjeka I., Bikandou B., Zekeng L., Takehisa J., Harada Y., Yamaguchi- Kabata Y., Taniguchi Y., Ido E., Kaptue L., M’pelle P., Parra H. J., Ikeda M., Hayami M., Miura T. (1999). Genetic diversity of HIV-1 group $\mathrm{M}$ from Cameroon and Republic of Congo. Arch Virol; 144, 2291-2311.

16. Nadler J. P., Wills T. S., Somboonwit C. (2003). Anaemia prevalence among HIV patients: antiretroviral therapy and other risk factors. Antiviral Therapy; 8, 1.

17. Nakanjako D., Kiragga A. N., Castelnuovo B., Kyabayinze D., Kamya M.R. (2011). Low prevalence of Plasmodium falciparum antigenaemia among asymptomatic HAART-treated adults in an urban cohort in Uganda. Malaria J.; 10:66-70.

18. Niama F. R., Toure-Kane C., Vidal N. (2006). HIV-1 subtypes and recombinants in the Republic of Congo. Infection Genetics and Evolution; 6, 337-343.

19. Van Geertruyden JP., D’Alessandro U. (2007). Malaria and HIV: a silent alliance. Trends in Parasitology; 23, 10, 466-467.

20. Omoregie R., Omokaro E. U., Palmer O. (2009). Prevalence of anemia among HIV-infected patients in Benin City, Nigeria. Tanzania journal of health Research; 11,1.

21. OMS. (2011). Résumé et points éssentiels. World Malaria report.

22. ONUSIDA.(2012).[online]http://www.unaids.org/en/dataanalysis/kno wyourresponse/countryprogressreports/2012countries/

23. Oumar A. A., Dao S., Goita D. (2009). Particularités de l'hémogramme de l'adulte atteint de VIH/SIDA en Afrique : à propos de 200 cas en milieu hospitalier de Bamako, Mali. Douvain Médical ; 128, 2 : 73-78. 
24. Rénia L., Potter S. M. (2006). Co-infection of malaria with HIV: an immunological perspective. Parasite Immunology; 28, 589-595.

25. Sanyaolu A. O., Fagbenro-Beyioku A. F., Oyibo W. A., Badaru O. S, Onyeabor O. S., Nnaemeka C. I. (2013). Malaria and HIV coinfection and their effect on hemoglobin levels from three health-care institutions in Lagos, southwest Nigeria. Afr Health Sci; 13, 2, 295300.

26. Satoguina J., Walther B., Drakeley C., Nwakanma D., Oriero E. C., Correa S., Corran P., Conway D. J, Walther M. (2009). Comparison of surveillance methods applied to a situation of low malaria prevalence at rural sites in The Gambia and Guinea Bissau. Malar J; $2,8,274$.

27. SEP/CNLS Congo (2009) Rapport [online] Available from: http://www.unaids.org/fr/dataanalysis/knowyourresponse/countrypro gressreports/2010countries/

28. Skinner-Adams T. S., McCarthy J. S., Gardiner D. L., Andrews K.T. (2008). HIV and malaria co-infection: interactions and consequences of chemotherapy. Trends in Parasitology; 24, 6, 264-271.

29. Volberding, P. 2008. Global HIV/AIDS Medicine. ISBN: 978-14160-2882-6. Section five: Prevention and Management in Resourcepoor Settings, Pages 747-753.

30. WHO. (2015). The global prevalence of anaemia in 2011 